\title{
Corrected Kriging update formulae for batch-sequential data assimilation
}

\author{
Clément Chevalier* \\ David Ginsbourger ${ }^{\dagger}$
}

March 28th, 2012

\section{Introduction}

Recently, a lot of effort has been paid to the efficient computation of Kriging predictors when observations are assimilated sequentially. In particular, Kriging update formulae enabling significant computational savings were derived in [1, 3, 2, Taking advantage of the previous Kriging mean and variance calculations helps avoiding a costly $(n+1) \times$ $(n+1)$ matrix inversion when adding one observation to the $n$ already available ones. In addition to traditional update formulae taking into account a single new observation, [2] also proposed formulae for the batch-sequential case, i.e. when $r>1$ new observations are simultaneously assimilated. However, the Kriging variance and covariance formulae given without proof in [2] for the batch-sequential case are not correct. In this paper we fix this issue and establish corrected expressions for updated Kriging variances and covariances when assimilating several observations in parallel.

\section{Kriging update formulae for the parallel case}

\section{1 notations and formulae}

In this section we give a counter-example for the Kriging variance update formula proposed in [2]. Let us first introduce the notations of this paper and recall these formulae. When $n$ observations, at locations $\mathbf{x}_{1}, \ldots, \mathbf{x}_{n} \in D$, of a real valued random field $Z$ indexed by $D \subset \mathbb{R}^{d}$ are available, [2] denotes respectively by $\hat{Z}(\mathbf{x})_{n}$ data and $\sigma_{n}^{2}(\mathbf{x})$ the Kriging mean and variance at $\mathbf{x} \in D$ based on these $n$ observations. The corresponding Kriging covariance is denoted by $\sigma_{n}:(\mathbf{x}, \mathbf{y}) \in D^{2} \rightarrow \sigma_{n}(\mathbf{x}, \mathbf{y})$. If $k>1$ new observations, at points $\mathbf{x}_{n+1}, \ldots, \mathbf{x}_{n+k}$, are available, the parallel Kriging update formulae given in [2]

*Institut de Radioprotection et de Sûreté Nucléaire (IRSN), Fontenay-aux-Roses, France

${ }^{\dagger}$ IMSV, Department of Mathematics and Statistics, University of Berne, Switzerland 
follow:

$$
\begin{aligned}
\hat{Z}(\mathbf{x})_{n+k \text { data }} & =\hat{Z}(\mathbf{x})_{n \text { data }}+\sum_{i=1}^{k} \lambda_{n+i \mid n+k}(\mathbf{x})\left(Z\left(\mathbf{x}_{n+i}\right)-\hat{Z}\left(\mathbf{x}_{n+i}\right)_{n \text { data }}\right) \\
\sigma_{n+k}^{2}(\mathbf{x}) & =\sigma_{n}^{2}(\mathbf{x})-\sum_{i=1}^{k} \lambda_{n+i \mid n+k}^{2}(\mathbf{x}) \sigma_{n}^{2}\left(\mathbf{x}_{n+i}\right) \\
\sigma_{n+k}(\mathbf{x}, \mathbf{y}) & =\sigma_{n}(\mathbf{x}, \mathbf{y})-\sum_{i=1}^{k} \lambda_{n+i \mid n+k}(\mathbf{x}) \lambda_{n+i \mid n+k}(\mathbf{y}) \sigma_{n}^{2}\left(\mathbf{x}_{n+i}\right)
\end{aligned}
$$

where $\lambda_{n+i \mid n+k}(\mathbf{x})$ denotes the Kriging weight of $Z\left(\mathbf{x}_{n+i}\right)$ when predicting $Z(\mathbf{x})$ relying on $n+k$ observations. In [2], these formulae are proven only for $k=1$. For $k>1$, they are quickly justified with an argument based on Pythagoras' theorem. In fact, the assumptions for using this theorem are not satisfied, as we will detail here. We now give a counter-example for Equation (2) and then propose fixed expressions.

\subsection{A counterexample for Equation (2)}

Let us consider for the sake of simplicity a case where $Z$ is a one-dimensional centered random process indexed by $D=[0,1]$, with covariance kernel $C(x, y):=\sigma_{0}(x, y)=$ $\min (x, y)$ (Wiener process, or Brownian Motion). Note that even though this process is non-stationary, Simple Kriging equations are applicable to it (Written in the general case of a non-stationary covariance kernel, as allowed in [2]'s results and notations). Assuming $n=0$ initial observations and $k=2$ new observations at the points $x_{1}=\frac{1}{2}$, $x_{2}=1$, the Kriging weights for a prediction at $x=\frac{3}{4}$ write:

$$
\left(\lambda_{1 \mid 2}(x), \lambda_{2 \mid 2}(x)\right)=\left(C\left(x, x_{1}\right), C\left(x, x_{2}\right)\right)\left(\begin{array}{ll}
C\left(x_{1}, x_{1}\right) & C\left(x_{1}, x_{2}\right) \\
C\left(x_{2}, x_{1}\right) & C\left(x_{2}, x_{2}\right)
\end{array}\right)^{-1}=\left(\frac{1}{2}, \frac{1}{2}\right)
$$

leading to a Kriging mean $\hat{Z}(\mathbf{x})_{n+k}$ data $=\frac{1}{2} Z\left(x_{1}\right)+\frac{1}{2} Z\left(x_{2}\right)$, and to a Kriging variance:

$$
\sigma_{2}^{2}(x)=C(x, x)-\left(\lambda_{1 \mid 2}(x), \lambda_{2 \mid 2}(x)\right)\left(\begin{array}{ll}
C\left(x_{1}, x_{1}\right) & C\left(x_{1}, x_{2}\right) \\
C\left(x_{2}, x_{1}\right) & C\left(x_{2}, x_{2}\right)
\end{array}\right)\left(\lambda_{1 \mid 2}(x), \lambda_{2 \mid 2}(x)\right)^{T}=\frac{1}{8}
$$

Now, using Eq. (21) would lead to

$$
\begin{aligned}
\sigma_{2}^{2}(x) & =\sigma_{0}^{2}(x)-\lambda_{1 \mid 2}^{2}(x) \sigma_{0}^{2}\left(x_{1}\right)-\lambda_{2 \mid 2}^{2}(x) \sigma_{0}^{2}\left(x_{2}\right) \\
& =\frac{3}{4}-\frac{1}{4} \frac{1}{2}-\frac{1}{4}=\frac{3}{8}
\end{aligned}
$$

and we see that the two expressions do not match. More precisely, the conditional variance obtained by using Eq. (2) is too large, as if the contribution of $x_{1}$ and $x_{2}$ to decreasing the variance would have been underestimated. As we will see now, this is indeed the case, and the missing part is related to conditional covariances. 


\subsection{Corrected Kriging variance and covariance update formulae}

In this section, we establish corrected update formulae that may be used in the batchsequential case instead of the formulae (21) and (3). To improve the readability of the properties and their proofs, we adopt the following simplified notations:

- $\mathbf{X}_{\text {old }}:=\left\{\mathbf{x}_{1}, \ldots, \mathbf{x}_{n}\right\}$, and $\mathbf{X}_{\text {new }}:=\left\{\mathbf{x}_{n+1}, \ldots, \mathbf{x}_{n+k}\right\}$,

- $\mathbf{Z}_{\mathrm{old}}:=\left(Z\left(\mathbf{x}_{1}\right), \ldots, Z\left(\mathbf{x}_{n}\right)\right)$, and $\mathbf{Z}_{\text {new }}:=\left(Z\left(\mathbf{x}_{n+1}\right), \ldots, Z\left(\mathbf{x}_{n+k}\right)\right)$,

- $\lambda_{\text {new }, \text { old }}(\mathbf{x}):=\left(\lambda_{1 \mid n+k}(\mathbf{x}), \ldots, \lambda_{n \mid n+k}(\mathbf{x})\right)^{T}$,

- $\boldsymbol{\lambda}_{\text {new,new }}(\mathbf{x}):=\left(\lambda_{n+1 \mid n+k}(\mathbf{x}), \ldots, \lambda_{n+k \mid n+k}(\mathbf{x})\right)^{T}$,

- $\sigma_{\text {old }}^{2}(\mathbf{x}):=\sigma_{n}^{2}(\mathbf{x}), \sigma_{\text {new }}^{2}(\mathbf{x}):=\sigma_{n+k}^{2}(\mathbf{x})$, and similarly for the conditional covariances.

- The conditional covariance matrix of $\mathbf{Z}_{\text {new }}$ knowing $\mathbf{Z}_{\text {old }}$ is denoted by $\Sigma_{\text {new }}$,

For conciseness and coherence, $\hat{Z}(\mathbf{x})_{n}$ data and $\hat{Z}(\mathbf{x})_{n+k}$ data are also denoted by $\hat{Z}(\mathbf{x})_{\text {old }}$ and $\hat{Z}(\mathbf{x})_{\text {new }}$, respectively. The corrected update formulae are given below:

Proposition 1. (Corrected Kriging update equations for the parallel case)

$$
\begin{aligned}
\hat{Z}(\mathbf{x})_{\text {new }} & =\hat{Z}(\mathbf{x})_{\text {old }}+\boldsymbol{\lambda}_{\text {new,new }}(\mathbf{x})^{T}\left(\mathbf{Z}_{\text {new }}-\hat{Z}\left(\mathbf{X}_{\text {new }}\right)_{\text {old }}\right) \\
\sigma_{\text {new }}^{2}(\mathbf{x}) & =\sigma_{\text {old }}^{2}(\mathbf{x})-\boldsymbol{\lambda}_{\text {new,new }}(\mathbf{x})^{T} \Sigma_{\text {new }} \boldsymbol{\lambda}_{\text {new }, \text { new }}(\mathbf{x}) \\
\sigma_{\text {new }}(\mathbf{x}, \mathbf{y}) & =\sigma_{\text {old }}(\mathbf{x}, \mathbf{y})-\boldsymbol{\lambda}_{\text {new, new }}(\mathbf{x})^{T} \Sigma_{\text {new }} \boldsymbol{\lambda}_{\text {new, new }}(\mathbf{y})
\end{aligned}
$$

The proofs above use a Gaussian assumption on the field $Z$, enabling a convenient interpretation of the Kriging mean and variance in terms of conditional expectation and variance. A key property is that even though the conditional distribution interpretation doesn't hold in non-Gaussian cases, the formulae for the Kriging weights remain valid whatever the assumed square-integrable distribution for the field; we are just using here that best linear prediction and conditioning coincide in the Gaussian case. Furthermore, the results hold as well for the cases of Ordinary and Universal Kriging (written in terms of covariances, for a square-integrable field, not with variograms), relying on their well-known Bayesian construction with an improper prior on the trend coefficient(s).

Proof. The first equation follows from an application of the law of total expectation:

$$
\begin{aligned}
\hat{Z}(\mathbf{x})_{\text {old }} & =\mathbb{E}\left[Z(\mathbf{x}) \mid \mathbf{Z}_{\text {old }}\right] \\
& =\mathbb{E}\left[\mathbb{E}\left[Z(\mathbf{x}) \mid \mathbf{Z}_{\text {old }}, \mathbf{Z}_{\text {new }}\right] \mid \mathbf{Z}_{\text {old }}\right] \\
& \left.=\mathbb{E}\left[\boldsymbol{\lambda}_{\text {new }, \text { old }}(\mathbf{x})^{T} \mathbf{Z}_{\text {old }}+\boldsymbol{\lambda}_{\text {new,new }}(\mathbf{x})^{T} \mathbf{Z}_{\text {new }}\right] \mid \mathbf{Z}_{\text {old }}\right] \\
& =\boldsymbol{\lambda}_{\text {new }, \text { old }}(\mathbf{x})^{T} \mathbf{Z}_{\text {old }}+\boldsymbol{\lambda}_{\text {new,new }}(\mathbf{x})^{T} \hat{Z}\left(\mathbf{X}_{\text {new }}\right)_{\text {old }} \\
& =\boldsymbol{\lambda}_{\text {new ,old }}(\mathbf{x})^{T} \mathbf{Z}_{\text {old }}+\boldsymbol{\lambda}_{\text {new,new }}(\mathbf{x})^{T} \mathbf{Z}_{\text {new }}-\boldsymbol{\lambda}_{\text {new }, \text { new }}(\mathbf{x})^{T} \mathbf{Z}_{\text {new }}+\boldsymbol{\lambda}_{\text {new }, \text { new }}(\mathbf{x})^{T} \hat{Z}\left(\mathbf{X}_{\text {new }}\right)_{\text {old }} \\
& =\hat{Z}(\mathbf{x})_{\text {new }}-\boldsymbol{\lambda}_{\text {new,new }}(\mathbf{x})^{T}\left(\mathbf{Z}_{\text {new }}-\hat{Z}\left(\mathbf{X}_{\text {new }}\right)_{\text {old }}\right)
\end{aligned}
$$


Similarly, using the law of total variance delivers:

$$
\begin{aligned}
\sigma_{\text {old }}^{2}(\mathbf{x}) & =\operatorname{Var}\left[Z(\mathbf{x}) \mid \mathbf{Z}_{\text {old }}\right] \\
& =\mathbb{E}\left[\operatorname{Var}\left[Z(\mathbf{x}) \mid \mathbf{Z}_{\text {old }}, \mathbf{Z}_{\text {new }}\right] \mid \mathbf{Z}_{\text {old }}\right]+\operatorname{Var}\left[\mathbb{E}\left[Z(\mathbf{x}) \mid \mathbf{Z}_{\text {old }}, \mathbf{Z}_{\text {new }}\right] \mid \mathbf{Z}_{\text {old }}\right] \\
& =\operatorname{Var}\left[Z(\mathbf{x}) \mid \mathbf{Z}_{\text {old }}, \mathbf{Z}_{\text {new }}\right]+\operatorname{Var}\left[\boldsymbol{\lambda}_{\text {new }, \text { old }}(\mathbf{x})^{T} \mathbf{Z}_{\text {old }}+\boldsymbol{\lambda}_{\text {new,new }}(\mathbf{x})^{T} \mathbf{Z}_{\text {new }} \mid \mathbf{Z}_{\text {old }}\right] \\
& =\sigma_{\text {new }}^{2}(\mathbf{x})+\boldsymbol{\lambda}_{\text {new,new }}(\mathbf{x})^{T} \sum_{\text {new }} \boldsymbol{\lambda}_{\text {new,new }}(\mathbf{x})
\end{aligned}
$$

which proves the second equation. The third equation comes with the same method, using the law of total covariance.

Proposition 2. (Kriging weights expressed in terms of conditional covariance)

$$
\Sigma_{\text {new }} \boldsymbol{\lambda}_{\text {new, new }}(\mathbf{x})=\sigma_{\text {old }}\left(\mathbf{X}_{\text {new }}, \mathbf{x}\right)
$$

Corollary 1. (Corrected Kriging update equations in terms of conditional covariance)

$$
\begin{aligned}
\hat{Z}(\mathbf{x})_{\text {new }} & =\hat{Z}(\mathbf{x})_{\text {old }}+\sigma_{\text {old }}\left(\mathbf{X}_{\text {new }}, \mathbf{x}\right)^{T} \Sigma_{\text {new }}^{-1}\left(\mathbf{Z}_{\text {new }}-\hat{Z}\left(\mathbf{X}_{\text {new }}\right)_{\text {old }}\right) \\
\sigma_{\text {new }}^{2}(\mathbf{x}) & =\sigma_{\text {old }}^{2}(\mathbf{x})-\sigma_{\text {old }}\left(\mathbf{X}_{\text {new }}, \mathbf{x}\right)^{T} \Sigma_{\text {new }}^{-1} \sigma_{\text {old }}\left(\mathbf{X}_{\text {new }}, \mathbf{x}\right) \\
\sigma_{\text {new }}(\mathbf{x}, \mathbf{y}) & =\sigma_{\text {old }}(\mathbf{x}, \mathbf{y})-\sigma_{\text {old }}\left(\mathbf{X}_{\text {new }}, \mathbf{x}\right)^{T} \Sigma_{\text {new }}^{-1} \sigma_{\text {old }}\left(\mathbf{X}_{\text {new }}, \mathbf{y}\right)
\end{aligned}
$$

Proof. Using the orthogonal projection interpretation of the conditional expectation,

$$
\begin{aligned}
Z(\mathbf{x}) & =\mathbb{E}\left(Z(\mathbf{x}) \mid \mathbf{Z}_{\text {old }}, \mathbf{Z}_{\text {new }}\right)+\overbrace{Z(\mathbf{x})-\mathbb{E}\left(Z(\mathbf{x}) \mid \mathbf{Z}_{\text {old }}, \mathbf{Z}_{\text {new }}\right)}^{=: \varepsilon} \\
& =\boldsymbol{\lambda}_{\text {new }, \text { old }}(\mathbf{x})^{\top} \mathbf{Z}_{\text {old }}+\boldsymbol{\lambda}_{\text {new,new }}(\mathbf{x})^{\top} \mathbf{Z}_{\text {new }}+\varepsilon,
\end{aligned}
$$

with $\varepsilon$ centered, and independent of $\mathbf{Z}_{\text {old }}$ and $\mathbf{Z}_{\text {new }}$. Let us now calculate the conditional covariance between $Z(\mathbf{x})$ and $\mathbf{Z}_{\text {new }}$ knowing the observations $\mathbf{Z}_{\text {old }}$ :

$$
\begin{aligned}
\sigma_{\text {old }}\left(\mathbf{X}_{\text {new }}, \mathbf{x}\right) & :=\operatorname{cov}\left(\mathbf{Z}_{\text {new }}, Z(\mathbf{x}) \mid \mathbf{Z}_{\text {old }}\right) \\
& =0+\operatorname{cov}\left(\mathbf{Z}_{\text {new }}, \boldsymbol{\lambda}_{\text {new,new }}(\mathbf{x})^{\top} \mathbf{Z}_{\text {new }} \mid \mathbf{Z}_{\text {old }}\right)+\operatorname{cov}\left(\mathbf{Z}_{\text {new }}, \varepsilon \mid \mathbf{Z}_{\text {old }}\right) \\
& =\Sigma_{\text {new }} \boldsymbol{\lambda}_{\text {new }, \text { new }}(\mathbf{x})+\operatorname{cov}\left(\varepsilon, \mathbf{Z}_{\text {new }} \mid \mathbf{Z}_{\text {old }}\right)
\end{aligned}
$$

Noting that $\operatorname{cov}\left(\mathbf{Z}_{\text {new }}, \varepsilon \mid \mathbf{Z}_{\text {old }}\right)=\mathbf{0}$, the latter equation proves Proposition 2, Eqs. 8, 9 , and 9 of the corollary directly follow by plugging in Eq. 22 into Eqs. 4, 5, 6,

\subsection{Interpretation on the counter-example}

The main difference between Equations 2 and 5 is that Eq. 5 takes into account conditional Kriging covariances. Coming back to the counter example, Eq. 5 delivers:

$$
\begin{aligned}
\sigma_{2}^{2}(x) & =\sigma_{0}^{2}(x)-\lambda_{1 \mid 2}^{2}(x) \sigma_{0}^{2}\left(x_{1}\right)-\lambda_{2 \mid 2}^{2}(x) \sigma_{0}^{2}\left(x_{2}\right)-\mathbf{2} \lambda_{\mathbf{1} \mid \mathbf{2}}(\mathbf{x}) \lambda_{\mathbf{2} \mid \mathbf{2}}(\mathbf{x}) \sigma_{\mathbf{0}}\left(\mathbf{x}_{\mathbf{1}}, \mathbf{x}_{\mathbf{2}}\right) \\
& =\frac{3}{4}-\frac{1}{4} \frac{1}{2}-\frac{1}{4}-\mathbf{2} \frac{\mathbf{1}}{\mathbf{4}} \frac{\mathbf{1}}{\mathbf{2}}=\frac{1}{8}
\end{aligned}
$$


which is the correct variance, as obtained earlier with regular Simple Kriging equations. Note that Eq. 2 would be correct if the matrix $\Sigma_{\text {new }}$ were diagonal, which has no particular reason to happen in practical applications. The Pytagorean theorem invoked in [2], enabling to decompose a variance into a sum of (weighted) variances, cannot hence be applied in the general case because of non-independence between Kriging residuals.

\section{Conclusion}

In this paper we derived corrected Kriging update formulae, fixing the incorrect formulae 2 and 3 given in [2], where a part of the update involving conditional covariances had been neglected. A simple interpretation of the new update formulae is that they correspond to Simple Kriging equations, where the underling covariance kernel would be the conditional covariance kernel before update, i.e $\sigma_{\text {old }}(.,$.$) . These formulae enable$ important computational savings (avoiding a large matrix inversion) and may perfectly be adapted to further frameworks such as co-Kriging.

Acknowledgements: The authors would like to thank Dr. Julien Bect (Ecole Supérieure d'Electricité) for drawing their attention to the conditional covariance interpretation of Kriging weights in the non-parallel case. Clément Chevalier acknowledges support from IRSN and the ReDICE consortium, David Ginsbourger acknowledges support from the IMSV, University of Berne.

\section{References}

[1] R. J. Barnes and A. Watson. Efficient updating of Kriging estimates and variances. Mathematical geology, 24(1):129-133, 1992.

[2] X. Emery. The Kriging update equations and their application to the selection of neighboring data. Computational Geosciences, 13(1):211-219, 2009.

[3] H. Gao, J. Wang, and P. Zhao. The updated Kriging variance and optimal sample design. Mathematical Geology, 28:295-313, 1996. 